\title{
Hitlers Zweites Buch - Eine vergessene Quelle der Zeitgeschichte zu Hitlers außenpolitischen Ansichten
}

\author{
Sabine Viktoria Kofler \\ Kerngebiet: Zeitgeschichte \\ eingereicht bei: ao. Univ.-Prof. Dr. Thomas Albrich \\ eingereicht im Semester: SS 2016 \\ Rubrik: SE-Arbeit
}

\begin{abstract}
Hitler's Second Book. A forgotten document of Hitler's views on foreign policies

The following seminar paper takes a closer look at one of the lesser-known writings of Adolf Hitler. His second book came to the surface only through the work of historians in the 1950s. This paper is to present the discovery, the following publication of this manuscript and the public and scholarly reaction to it. Secondly, it will contemplate Hitler's thoughts and views on German foreign policy. Finally, it will discuss several theories on why this unfinished manuscript never came to be published until 1961 in the first place.
\end{abstract}

\section{Einleitung}

Die Existenz eines zweiten Buches von Adolf Hitler ist wohl nur einigen Fachleuten bewusst. Den Bekanntheitsgrad von "Mein Kampf" hat dieses zweite Werk seit seinem Erscheinen im Jahr 1961 in der breiten Öffentlichkeit nie erreicht. Bei der Lektüre des Manuskripts, dessen Niederschrift bereits 1929 erfolgte, wird allerdings schnell klar, dass darin kein völlig neues Gedankengut des Nationalsozialisten zur Sprache kommt. Vielmehr werden hier Hitlers ideologische und weltpolitische Ansichten ausgeführt, die es auch in "Mein Kampf" zu lesen gibt. Einen besonderen Schwerpunkt bildet in dem Schriftstück die Bündnispolitik der Nationalsozialisten, die zu der Zeit von dessen Entstehen grundlegend von der vorherrschenden Außenpolitik des Deutschen Reichs abwich. 
Das unveröffentlichte Manuskript lag jahrelang im Tresor des Franz-Eher-Verlags, von wo es 1945 zusammen mit anderen Akten in die USA überführt wurde. Erst Jahre später gelang es dem Historiker Gerhard L. Weinberg dieses einzigartige Dokument zu identifizieren und erstmals mit kritischem Kommentar und einer Einleitung versehen 1961 herauszugeben.' Das Institut für Zeitgeschichte in München nahm dieses HitlerManuskript schließlich 1995 in die Editionsreihe "Hitler. Reden, Schriften, Anordnungen" unter dem neuen Namen "Außenpolitische Standortbestimmungen nach der Reichstagswahl 1928" auf.2

In dieser Seminararbeit wird den Fragen nachgegangen, unter welchen Umständen das Manuskript wiederentdeckt wurde, warum Hitler es für nötig befand, ein weiteres Werk über nationalsozialistische Außenpolitik zu schreiben und wieso es schlussendlich nicht schon im Jahre seiner Entstehung herausgegeben wurde.

In einem ersten Überblick werden die Überlieferungsgeschichte und die Veröffentlichung des Manuskriptes näher untersucht werden. Die Reaktionen und Diskussionen um diese Edition sollen in einem weiteren Punkt anhand ausgewählter Rezensionen dargestellt werden. Ein kurzer Exkurs wird auch den ungewöhnlichen Werdegang der englischen Edition behandeln.

Im nächsten Teil der Arbeit werde ich den Inhalt der Quelle und seine Entstehungsgeschichte behandeln. Die Ursache und der Anlass für Hitlers zweites Werk sollen anhand einer Darstellung der Situation von 1928, insbesondere auch die Reichstagswahl in diesem Jahr, näher betrachtet werden. In diesem Hinblick spielte auch das vom Faschismus unterdrückte Südtirol eine Schlüsselrolle zur Entstehung der Schrift. Der letzte Punkt dieser Arbeit erörtert die Frage, wieso es schlussendlich nicht zu einer Veröffentlichung durch Hitler oder seinen Verlag kam.

Die Forschungsliteratur über Hitlers Zweites Buch ist überschaubar. Die ausführlichsten Informationen zu Fund und Überlieferung bietet der Herausgeber der Edition, Weinberg, selbst. Ansonsten widmen die Historiker diesem Manuskript höchstens einen kurzen Einschub, wo es ansonsten um die Bearbeitung des weitaus bekannteren Werkes "Mein Kampf", geht. Sven Felix Kellerhoff (2015) ist dabei mit einem eigenen Kapitel über Hitlers Zweitem Buch noch weitaus ausführlicher als die meisten. ${ }^{3}$ Dementsprechend ist sein Beitrag auch für diese Arbeit sehr wertvoll, denn er bildet eine gute Ergänzung zu Weinbergs eigenen Recherchen. Othmar Plöckinger (2011) und Antoine Vitkine (2015) hingegen widmen Hitlers Zweitem Buch nur wenige Seiten, bieten dafür aber neue Ein-

$1 \quad$ Hitlers Zweites Buch. Ein Dokument aus dem Jahr 1928. Eingeleitet und kommentiert von Gerhard L. Weinberg. Mit einem Geleitwort von Hans Rothfels, (Quellen und Darstellungen zur Zeitgeschichte 7), Stuttgart 1961. Alle Zitate, die in dieser Arbeit verwendet wurden, sind aus Weinbergs Edition entnommen.

2 Institut für Zeitgeschichte (Hrsg.), Außenpolitische Standortbestimmung nach der Reichstagswahl. Juni-Juli 1928 (Hitler. Reden, Schriften, Anordnungen Bd. 2-A), München 1995, siehe dazu auch: Othmar Plöckinger, Geschichte eines Buches: Adolf Hitlers „Mein Kampf“ 1922-1945, München 2011², S. 161.

3 Sven Felix Kellerhoff, "Mein Kampf“. Die Karriere eines deutschen Buches, Stuttgart 2015, S. 171-191. 
sichten, die Weinbergs Interpretationen aus den 1960er Jahren auf den aktuellen Stand der Forschung bringen. ${ }^{4}$

\section{Hitlers Zweites Buch}

\subsection{Entdeckung und Veröffentlichung}

Erste Gerüchte und Vermutungen über die Existenz eines zweiten Buches von Hitler gab es schon Anfang der 1950er Jahre. Der deutsche Historiker Hans Rothfels (18911976) ${ }^{5}$ schildert in einem Geleitwort zur Edition des zweiten Hitlerbuches, wie erste Hinweise an das Institut für Zeitgeschichte gelangten. Daraufhin erfolgten im Mai/Juni 1951 erste Nachforschungen des Historikers Hermann Mau in den USA, die aber erfolglos blieben. Damals wurde noch nach einem verschollenen Hitler-Manuskript aus dem Jahre 1935 gesucht. ${ }^{6}$ Der britische Historiker Hugh Redwald Trevor-Roper gab bei einem Vortrag in München 1959 an, der ein Jahr später in den Vierteljahrsheften für Zeitgeschichte erschien, Hitler habe ein zweites Buch über seine außenpolitischen Pläne bezüglich Frankreich und England 1924 verfasst. Dieses Buch soll laut TrevorRoper allerdings nicht mehr existieren.7 Trevor-Roper behauptet anschließend, Rudolf Heß habe von diesem zweiten Buch gewusst. Hitlers Pläne für ein Bündnis mit England, die in dieser Schrift ausgeführt wurden, seien für ihn sogar ein Anlass für seinen spektakulären Flug nach England 1941 gewesen. ${ }^{8}$ Weitere Hinweise auf ein zweites Hitlerbuch kamen in den späten 1950er Jahren von einer glaubwürdigen Quelle. Josef Berg, ehemaliger Mitarbeiter im Franz-Eher-Verlag, bezeugte in einem Brief vom 12. September 1958 an das Münchner Institut für Zeitgeschichte die Existenz dieses zweiten Hitler-Buches. In Bergs Brief gab es zwar einige Angaben, die sich bald als Gedächtnisfehler herausstellten, jedoch sonst durchaus richtige Aussagen, wie sich später zeigen sollte. Berg schilderte die strenge Geheimhaltung des Manuskriptes und seine Beschlagnahmung durch einen amerikanischen Offizier am Ende des Krieges. Daraufhin wurde Rothfels vom Institut beauftragt dieses Manuskript im Herbst 1958 in den USA zu suchen. Rothfels bat bei dieser Suche seinen ehemaligen Schüler Gerhard Ludwig Weinberg um Hilfe. ${ }^{9}$ Weinberg, 1928 in Deutschland geboren und mit seiner Familie 1940 nach Amerika emigriert, ${ }^{10}$ war zu diesem Zeitpunkt Associate Professor für Geschichte an der Michigan University. ${ }^{11}$ Weinberg schrieb wichtige Forschungsarbeiten zu Nazideutschland, beschäftigte sich auch intensiv mit Hitlers Außenpolitik und den Dokumenten der Nationalsozialisten, die nach dem Krieg aus Deutschland in die

4 Plöckinger, Geschichte eines Buches - Antoine Vitkine, Hitlers „Mein Kampf“. Geschichte eines Buches, Hamburg 2015.

$5 \quad$ Wolfgang Neugebauer, Rothfels, Hans, in: Neue Deutsche Biographie 22 (2005), S. 123-125 Onlinefassung: [https://www.deutsche-biographie.de/\%20pnd118749943.html], eingesehen 22.8.2016.

6 Rothfels, Geleitwort, in: Hitlers Zweites Buch, S. 7.

$7 \quad H u g h$ Redwald Trevor-Roper, Hitlers Kriegsziele, in: Vierteljahrshefte für Zeitgeschichte 8 (1960), Heft 2, S. 121-133, hier 133.

8 Ebd.

9 Rothfels, Geleitwort, S. 7.

10 Jahresberichte für Deutsche Geschichte, 22.12.2015, [http://jdgdb.bbaw.de/cgi-bin/jdg?lang=de\&t_ $i d n=\% 28 D E-588 \% 29105818925]$, eingesehen 22.8.2016.

11 Rothfels, Geleitwort, S. 7. 
USA überführt worden waren. ${ }^{12}$ Für diese Recherche war Weinberg also ein geeigneter erster Ansprechpartner. In der Tat hatte auch Weinberg schon von sich aus Nachforschungen über dieses verschollene Hitler-Dokument angestellt und es tatsächlich in den Akten, die vor ihrer Rückgabe nach Deutschland auf Mikrofilm aufgenommen wurden, gefunden. ${ }^{13}$ Im Sommer 1958 fand Weinberg im amerikanischen Nationalarchiv in der Abteilung II. Weltkrieg ein mit Schreibmaschine getipptes Manuskript von 324 Seiten zur deutschen Außenpolitik. Anhand eines beiliegenden Beschlagnahmeprotokolls konnte seine Identität zweifelsfrei festgestellt werden. In diesem in den 1940ern erstellten Protokoll wurde vermerkt, dass Josef Berg, ehemaliger technischer Leiter des Verlags, das Manuskript eines angeblich unveröffentlichten Werks von Hitler an einen US-Offizier übergab, welches „vor über 15 Jahren geschrieben und in einen Tresor geschlossen" wurde. ${ }^{14}$ Somit bestätigte sich Bergs Aussage in seinem Brief an das Institut und Weinberg galt als der Entdecker des zweiten Hitlerbuches.

Josef Berg hatte 1935 die Abteilung Buchverlag im Eher-Verlag übernommen und damit auch das unveröffentlichte Manuskript, welches er im Tresor und später im Luftschutzkeller des Verlags aufbewahrte. Außer dem Verlagsexemplar soll sich noch eine weitere Kopie auf dem Obersalzberg befunden haben, die allerdings wahrscheinlich gegen Ende des Krieges mit anderen wichtigen Dokumenten verbrannt wurde. ${ }^{15}$ Für die Existenz dieser zweiten Kopie spricht die Tatsache, dass die Seiten 240-324 des Manuskripts Schreibmaschinendurchschläge sind. Bei der Ordnung der Blätter muss es zu einer Verwechslung des Originals und der Kopie gekommen sein, womit feststeht, dass es mindestens eine Kopie gegeben hat. ${ }^{16}$

Im Mai 1945 übergab Berg das Manuskript einem amerikanischen Offizier. Es wurde zusammen mit anderen Akten in die USA überführt, wo es jedoch fälschlicherweise als Entwurf zu "Mein Kampf" aufgenommen wurde. ${ }^{17}$ Weinberg konnte es dann 1958 aufgrund des Begleitprotokolls, der Aussagen von Josef Berg und des Inhalts des Manuskripts richtig als Entwurf für ein zweites Hitlerbuch identifizieren. In Zusammenarbeit mit dem Institut für Zeitgeschichte in München wurde es von Weinberg kritisch kommentiert und eingeleitet in der Reihe "Quellen und Darstellungen zur Zeitgeschichte" 1961 veröffentlicht.

Dabei stellte sich auch den damaligen Herausgebern die Frage des Urheberrechts. Der Franz-Eher-Verlag ging nach dem Krieg an die bayerische Regierung über und somit auch die Rechte von "Mein Kampf“. Allerdings hatte Hitler nie einen Vertrag mit dem Verlag über dieses zweite Manuskript angefertigt und die Rechte gingen in diesem Fall an Hitlers Erben. Das Münchner Institut kaufte diese Verlagsrechte kurzerhand den Erben ab, um mögliche Probleme mit der Regierung wegen einer Veröffentlichung zu

12 Rothfels, Geleitwort, S. 7-8.

13 Daniel Johnson, Revealed: the amazing story behind Hitler's second book, in: The Telegraph 25.9.2003, [http:// www.telegraph.co.uk/culture/books/3603289/Revealed-the-amazing-story-behind-Hitlers-second-book.html], eingesehen 22.8.2016.

14 Weinberg, Anlage, in: Hitlers Zweites Buch, S. 225.

15 Kellerhoff, „Mein Kampf", S. 189.

16 Weinberg, Einleitung, in: Hitlers Zweites Buch, S. 15-16.

17 Kellerhoff, "Mein Kampf", S. 189. 
verhindern. ${ }^{18}$ Auf die Gründe für die Veröffentlichung kommen Weinberg und Rothfels ebenfalls zu sprechen. Gab es denn Bedenken und sollte die Verbreitung von nationalsozialistischem Gedankengut nicht unterbunden werden? Das Argument, der Neonazismus könnte sich durch diese Edition ausbreiten, wiesen beide Historiker entschlossen zurück, da jeder, der sich mit der Ideologie Hitlers auseinandersetzen wollte, die gleichen Gedanken auch problemlos in "Mein Kampf“ nachlesen könne. Weinberg meint zudem, dass Hitler und die NSDAP im Deutschland der 1930er Jahre nicht durch die massenhafte Lektüre von "Mein Kampf" großgeworden seien. ${ }^{19}$ Für eine kritische Lektüre sei es daher wichtig, Hitlers Zweites Buch mit einer Erläuterung des historischen Kontextes durch Historiker der Öffentlichkeit und Forschung zugänglich zu machen. Auch wenn das Buch für Hitlerkenner wenig Neues bietet, sei dieses Schriftstück doch außergewöhnlich in seiner Existenz. Rothfels weist vor allem auf die Bedeutung dieses Dokumentes als Quelle für die Zeitgeschichte und die Verantwortung der Wissenschaft hin:

„Eine ihrer Verantwortung bewußte Wissenschaft kann sich nicht darauf einlassen, das Vergessenwollen [sic!] zu unterstützen, das immer wieder sich anmeldet, und ist nicht berechtigt, die brutale Sprache, die in diesem Zweiten Buch zum Teil noch schriller ist als in,Mein Kampf', ungehört verhallen zu lassen." ${ }^{20}$

Noch weitere Gründe sprechen für eine Auseinandersetzung mit dieser Quelle. Rothfels glaubt die Tatsache, dass Hitler selbst, aus welchen Gründen auch immer, das Buch nicht veröffentlichte, schon eine gewisse Begründung für eine derzeitige Publikation sei. ${ }^{21}$ Weinberg gibt noch weitere Beweggründe an, warum die Veröffentlichung dieses Werks wichtig sei: Obwohl Hitler in diesem Manuskript viele altbekannte Standpunkte vorbringt, die er auch schon in "Mein Kampf" behandelt hatte, sei dieser Entwurf doch eine wertvolle Quelle für die Kontinuität und Entwicklungen (oder das Fehlen einer solchen Entwicklung) seiner Gedanken. Besonders da die Zeitspanne zwischen der Niederschrift von "Mein Kampf“ und Hitlers Machtergreifung bis dahin noch wenig beachtet und erforscht worden war. ${ }^{22}$

Ein weiterer bedeutsamer Punkt ist auch der biographische Wert der Quelle, denn zum Zeitpunkt der Entstehung dieses Buches war Hitler alles andere als der machtvolle autoritäre Diktator, der sich im Kollektivbewusstsein späterer Generationen festgesetzt hatte. Im Gegenteil, nach der WahIniederlage von 1928, sah die politische Zukunft der Partei und ihres Führers nicht sehr erfolgsversprechend aus. Als letzten Punkt nennt Weinberg noch den Bezug zur eigenen Gegenwart, den diese Quelle hatte. In den frühen 1960er Jahren waren die Alltagsprobleme und politischen Geschehnisse für die Bevölkerung von größerer Bedeutung als die unmittelbare Vergangenheit. Weinberg sah darin die Gefahr, dass viele Quellen übersehen und die Bereitschaft sich mit Hitler als Person und Symbol auseinanderzusetzen, abnehmen würde. Hitlers Taten aber

18 Johnson, Revealed.

19 Weinberg, Einleitung, S. 38.

20 Rothfels, Geleitwort, S. 9

21 Ebd

22 Weinberg, Einleitung, S. 38. 
wirkten auch noch lange nach Ende von Nazideutschland in der Welt nach. ${ }^{23}$ Deshalb war laut Weinberg der folgende Anspruch gegeben:

„Nur vertieftes Verständnis des Bösen kann der Menschheit helfen, mit diesen Folgen moralisch fertig zu werden; möge die Veröffentlichung hierzu beitragen." 24

Die Herausgeber des Zweiten Buches standen offensichtlich vor demselben Dilemma bezüglich der Frage eines möglichen Erlöses durch den Verkauf von Hitlers Zweitem Buch wie die Herausgeber der Neuauflage von "Mein Kampf". Daher wurde festgelegt, „um auch die leiseste, wenngleich unwahrscheinliche Mißdeutung der Publikation auszuschließen"25, einen möglichen Reinertrag an Opfer der nationalsozialistischen Verfolgung zu spenden.

Im drastischen Gegensatz zur kritisch kommentierten Neuauflage von "Mein Kampf" - mittlerweile ein wahrer Bestseller ${ }^{26}$ - löste die Bearbeitung und Veröffentlichung von Hitlers Zweitem Buch außerhalb der Geschichtswissenschaft jedoch weit weniger Schlagzeilen in den Medien aus.

\subsection{Rezensionen}

Nach der Veröffentlichung erschien am 4. August 1961 ein kurzer Artikel in der Zeit unter dem Autorenkürzel H.K. Wahrscheinlich handelte es sich dabei um Heinz Stuckmann. ${ }^{27}$ Darin sieht auch Stuckmann den Quellenwert, den dieses Dokument für die Historiker hat und betont, dass es ebenso für die Gesellschaft wertvoll sei:

„In der Tat war es auch unter, politisch-pädagogischem Gesichtspunkt geboten, dieses Manuskript, das sein Verfasser aus guten Gründen unter Verschluß gehalten hat, ans Licht der Öffentlichkeit zu ziehen. Denn schlagender und überzeugender kann keine Entlarvung Hitlers sein, als diese, die er selber hier bietet." $^{\text {28 }}$

Stuckmann spielte in diesem Zusammenhang auf die Meinung hartnäckiger Hitlerverteidiger an, die behaupteten, Hitler habe stets nur die Revision des Versailler Vertrages und das Selbstbestimmungsrecht für alle Deutschen gewollt. In diesem Buch jedoch legte Hitler seine außenpolitischen Pläne schon 1928 offen dar. Mit Hitlers steter Betonung auf die Notwendigkeit eines „Lebensraums“ im Osten für die deutsche Bevölkerung und auch der Bereitschaft dafür in den Krieg zu ziehen, ist dieses Argument für Stuckmann entkräftet. Auch die Verantwortung für den Ausbruch des Krieges sieht der

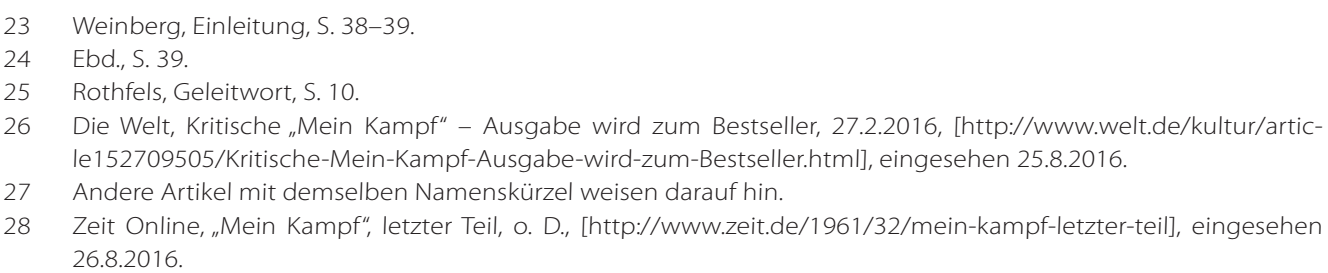

28 Zeit Online, "Mein Kampf“, letzter Teil, o. D., [http://www.zeit.de/1961/32/mein-kampf-letzter-teil], eingesehen 26.8.2016. 
Autor des Artikels damit klar Hitler zugewiesen.. ${ }^{29}$ Die besondere Hervorhebung der Kriegsschuld bei Stuckmann ist wohl eine Reaktion auf die hochkontroverse Debatte um den britischen Historiker Alan J.P. Taylor, der zu dieser Zeit für großen Wirbel mit der These, Hitler trage nicht die Alleinschuld am Ausbruch des Zweiten Weltkrieges, sorgte. Von den westdeutschen Historikern wurden Taylors Arbeit und seine provokanten ÄuBerungen zu den Ursachen des Zweiten Weltkriegs weitgehend negativ und skeptisch aufgenommen. ${ }^{30}$ In diesem Sinne ist wohl auch der explizite Aufruf von Stuckmann in seinem Artikel zu verstehen, der eine konkrete Gefahr in „Darstellungen à la Taylor über die Verantwortlichkeit für den Zweiten Weltkrieg“ sah. ${ }^{31}$ Auch Hitlers außenpolitische Pläne bezüglich Russland werden laut Stuckmann in Hitlers Zweitem Buch eindeutig dargelegt. Der Osten war für Hitler "Lebensraum“ und anderweitige Aussagen oder außenpolitische Handlungen bezüglich der Sowjetunion nach seiner Machtergreifung enthüllten sich als bloße Propaganda. ${ }^{32}$

Martin Broszat geht in seinen „Betrachtungen zu Hitlers Zweitem Buch“33 (1961) gründlicher auf den Inhalt des Hitlermanuskripts ein. Er führt Hitlers Weltanschauung, seine einseitigen und unsachlichen Lösungsansätze zu Fragen der deutschen Außen- und Innenpolitik an und zeigt dabei auf, wie sich Hitler oftmals in Widersprüche verstrickte und seine unlogischen und von Wunschdenken durchzogenen Ideen darlegte. Broszat fasst in seinen Betrachtungen zusammen, dass Hitler, wie schon vorher in "Mein Kampf" der Rhetorik und Demagogie verpflichtet blieb. Sein schriftliches Werk sei dabei "weit von logischer Systematik und Gliederung entfernt".34 Die Edition stellt nichtsdestoweniger eine gute Quellenerweiterung für Hitlers außenpolitische Vorstellungen dar, die in "Mein Kampf" vergleichsweise kurzgehalten waren. Die Aussagen im Buch stützten zudem Hitlers Gedankengänge, beispielsweise zur Bündnispolitik, die er schon in "Mein Kampf" vorgebracht hatte. Broszat ist aber auch der Meinung, dass "Mein Kampf" bis 1945 zwar millionenfach verkauft wurde, allerdings kaum gelesen und von Hitlers Gegnern nicht ernst genommen wurde. ${ }^{35}$ Erst nach Kriegsende hätten sich Historiker kritischer mit "Mein Kampf" auseinandergesetzt, dabei indes lediglich nach Zitaten und Belegen für Hitlers Pläne gesucht. Hitlers Wesen selbst und sein Denken seien bei diesen Arbeiten jedoch nicht gründlich genug analysiert und untersucht worden. ${ }^{36}$

30 Der Spiegel, Adolf Hitler - Weder Held noch Schurke?, o. D., [http://www.spiegel.de/spiegel/print/d-43367625. html], eingesehen 26.8.2016.

31 Zeit Online, ,Mein Kampf".

32 Ebd

33 Martin Broszat, Betrachtungen zu Hitlers Zweitem Buch, in: Vierteljahrshefte für Zeitgeschichte 9 (1961), Heft 4, S. 417-429. 
Waldemar Bessons Rezension zu "Hitlers Zweitem Buch" erschien 1963 in der "Historischen Zeitschrift“. ${ }^{37}$ Der Autor kommt schon zu Beginn auf den „Wirbel politischer Verdächtigungen“38, in denen das Erscheinen dieses Buches geriet, zu sprechen. Es hätte Vorwürfe hinsichtlich eines ",angeblichen Neonazismus in der Bundesrepublik“39 gegeben nach Veröffentlichung der Edition von westlicher wie auch östlicher Seite. Allerdings waren sich das IfZ und die Herausgeber durchaus ihrer politischen Verantwortung bewusst, die sie mit der Herausgabe von Hitlers Zweitem Buch auf sich nahmen, denn "gerade eine wissenschaftliche Edition mußte jede sensationelle Aufbauschung verhindern"40. Besson hob besonders die Aussage der Herausgeber hervor, jegliche Reinerlöse der 228 Seiten zählenden Edition bei einem Kaufpreis von 19,80 DM an die Opfer des Nationalsozialismus zu spenden. ${ }^{41}$ Wie schon die Rezensionen vor ihm, unterstrich auch Besson den wissenschaftlichen Quellenwert von Hitlers Zweitem Buch, vor allem für eine Zeitperiode, welche die Forschung bisher aus Mangel an Quellen wenig bearbeitet habe. Eine Edition von Hitlers unveröffentlichtem Manuskript zeichnet zudem ein genaueres Bild von Hitlers außenpolitischen Vorstellungen und "schließt für das Prinzipielle eine Lücke vor allem dadurch, daß sie uns nachdrücklich die Konstanz in Hitlers Gedankengängen bezeugt"42.

Jahrzehnte später nahm in einem Online-Blog 2009 der SPD-Politiker Mathias Brodkorb Stellung zu Hitlers Zweitem Buch. ${ }^{43}$ Schon durch den Titel des Beitrags, "Hitlers Zweites Buch. Mit den europäischen Nationen gegen amerikanische Globalisierung?" ist erkenntlich, dass sich der Autor mit gänzlich anderen Aspekten auseinandersetzt als die Rezensionen vor ihm. Brodkorb widerspricht zunächst den Aussagen von Rothfels und Weinberg, die Sprache in diesem zweiten Hitlerbuch sei zum Teil noch schriller und brutaler als in "Mein Kampf". Antisemitische Hasstiraden und Anfeindungen gegen vermeintliche Gegner würden sich nicht wesentlich von jenen in "Mein Kampf" unterscheiden. ${ }^{44}$ Außerdem nahmen Hitlers Pläne für eine zukünftige Auseinandersetzung mit den USA in diesem zweiten Buch konkretere Züge an. Amerika würde für Europa und Deutschland zu einem gefährlichen Konkurrenten auf dem Weltmarkt werden und Hitler hätte, so Brodkorb, zumindest ansatzweise eine Vision eines Bundes von freiheitlichen Nationen Europas, die den Vereinigten Staaten entgegentreten würden. ${ }^{45}$ Solche Aussagen könnten sich durchaus Rechtsextremisten von heute zunutze machen, die bekanntlich der globalisierten Welt und den USA feindlich eingestellt sind. Zuletzt spricht Brodkorb noch von der bizarren Situation, dass Hitlers Zweites Buch

37 Waldemar Besson, Rezension zu: Hitlers zweites Buch. Ein Dokument aus dem Jahre 1928. Eingeleitet und kommentiert von Gerhard L. Weinberg. Mit einem Geleitwort von Hans Rothfels, Stuttgart 1961, in: Historische Zeitschrift, 197 (1963), Heft 3, S. 670-673, [http://www.jstor.org/stable/27613666], eingesehen 26.5.2016.

43 Mathias Brodkorb, Hitlers Zweites Buch. Mit den europäischen Nationen gegen amerikanische Globalisierung?, Störungsmelder, Blogeintrag vom 21.5.2009, [http://blog.zeit.de/stoerungsmelder/2009/05/21/hitlers-zweitesbuch-mit-den-europaischen-nationen-gegen-amerikanische-globalisierung_1077], eingesehen 29.8.2016. 
bereits seit 1961 durch einen deutschen Verlag veröffentlicht wurde, eine Neuauflage von Hitlers "Mein Kampf" jedoch von der bayerischen Staatsregierung weiterhin abgelehnt wurde. Dabei sei Hitlers Werk heutzutage für Interessierte in fast allen Sprachen der Welt im Internet frei zugänglich. ${ }^{46}$

\subsection{Englische Edition}

Weinberg plante nach der deutschen Edition auch eine englische Übersetzung von Hitlers Zweitem Buch. Dazu kam es jedoch erst vierzig Jahre nach der deutschen Ausgabe. In einem Interview mit der britischen Zeitung The Telegraph, welches anlässlich der Veröffentlichung dieser englischen Ausgabe 2003 erschien, schildert Weinberg, warum eine englische Übersetzung erst so spät auf dem Buchmarkt kam. ${ }^{47}$ Weinberg war mit der unangenehmen Tatsache konfrontiert, dass es ein englisches Plagiat seiner Edition gab, die unter dem Titel "Hitler's Secret Book" 1962 veröffentlicht wurde. Anstatt das Originalmanuskript als Vorlage für eine möglichst wortgetreue Übersetzung heranzuziehen, wurde Weinbergs deutsche Ausgabe übersetzt. Sie gilt deshalb in Historikerfachkreisen als unzuverlässig. Da Weinberg, wie er selbst sagte, kein Geld für den Verkauf der Edition annahm, konnte er nach geltendem Recht die Herausgeber der englischen Raubkopie nicht verklagen, da er ja keinen finanziellen Verlust erlitten hatte. ${ }^{48}$ Im Nachhinein stellte sich also der Verzicht auf die Einnahmen durch den Buchverkauf als ein ungewollter Rückschlag für den Historiker heraus.

Nach Erscheinen dieser Kopie konnte Weinberg keinen englischen Verlag finden, der an einer weiteren Ausgabe interessiert war. Erst 2003 erschien im Enigma Verlag unter dem Titel "Hitler's Second Book. The Unpublished Sequel to Mein Kampf" unter Mitarbeit von Weinberg eine vollständige, wissenschaftlich kommentierte englische Ausgabe. $^{49}$

In den Rezensionen wird allgemein kritisiert, dass Hitlers Zweites Buch, bis auf Ausnahmen aus Historikerkreisen, kaum beachtet wurde, obwohl es für Hitlers außenpolitische Vorstellungen eine weitaus bessere Quelle bieten würde, als "Mein Kampf"50. Auch in der deutschen Öffentlichkeit wurde das Erscheinen der Edition von Weinberg 1961 eher verhalten zur Kenntnis genommen. Die Bevölkerung interessierte sich zu dieser Zeit anscheinend mehr für die turbulenten politischen Tagesereignisse. ${ }^{51}$

Mathias Brodkorb, Hitlers Zweites Buch. Mit den europäischen Nationen gegen amerikanische Globalisierung?, Störungsmelder, Blogeintrag vom 21.5.2009, [http://blog.zeit.de/stoerungsmelder/2009/05/21/hitlers-zweitesbuch-mit-den-europaischen-nationen-gegen-amerikanische-globalisierung_1077], eingesehen 29.8.2016.

47 Johnson, Revealed.

48 Ebd.

49 Enigma Books, O. D., [http://www.enigmabooks.com/store/catalog/moreinfo.php?id=32], eingesehen 29.8.2016.

50 Richard Overy, Mein Kampf. The Sequel, in: The Guardian, 1.7.2003, [https://www.theguardian.com/books/2003/ jul/01/news], eingesehen 30.8.2016 - Nitzan Lebovic, Hitler in a Post-Ideological Age. The Sequel, in: H-German. H-Net Reviews, 2006, [http://www.h-net.org/reviews/showrev.php?id=11363], eingesehen 30.8.2016. 


\section{Hitlers Manuskript zur deutschen Außenpolitik}

\subsection{Inhalt und Entstehung}

Das überlieferte Manuskript von Hitler weist einige Leerstellen auf und nicht alle Kapitel scheinen vollständig abgeschlossen zu sein. Josef Berg behauptete in seinem Brief an das IfZ, Hitler habe sein zweites Buch Max Amann, dem Leiter des Franz-Eher Verlags, diktiert. ${ }^{52}$ Kellerhoff hingegen vermutet, er habe es, wie schon „Mein Kampf“, einer Privatsekretärin in die Schreibmaschine diktiert. ${ }^{53}$

Das Manuskript von 324 Seiten umfasst 15 Kapitel, ein Vor- sowie ein Schlusswort. Die Kapiteleinteilung hat Weinberg aus Vermerken entnommen, die Titelüberschriften selbst stammen aber von ihm. ${ }^{54}$ Den ersten Kapiteln widmet Hitler dem Thema des „Lebenskampfes" der deutschen Bevölkerung. Er ist davon überzeugt, dass es für das Überleben der Deutschen unvermeidlich sei mehr Land für die Nahrungsmittelversorgung zur Verfügung zu haben. Diesen „Lebensraum“ sieht Hitler für den eigentlichen Grund einen gerechtfertigten Krieg zu führen. Daraus schlussfolgert er: „Aus der Not des Krieges erwächst dann das Brot des Friedens." ${ }^{45}$ Alternativen zur Kriegsführung, wie Neutralität oder Wirtschaftswachstum lehnt Hitler dagegen strikt ab und sieht sie als undurchführbar an. Das außenpolitische Handeln eines deutschen Staates muss nach seiner Vorstellung deshalb allein auf das Erreichen dieses "Lebensraums" hinarbeiten.

In den folgenden Kapiteln spricht Hitler von Fehlern und dem Versagen der Regierung diese Raumpolitik durchzuführen, diskutiert über die Niederlage im Ersten Weltkrieg und sieht auch in Amerika einen neuen Konkurrenten auf der Bühne der Weltpolitik. Die Juden und die Gefahr eines "Weltjudentums“56 kommen in Hitlers Zweitem Buch zwar auch vor, allerdings nehmen sie eine relativ untergeordnete Stellung ein. Auf seine eigentlichen außenpolitischen Ziele und die Bündnispolitik, die ihm vorschwebte, kommt Hitler erst im letzten Teil zu sprechen. ${ }^{57}$ In "Mein Kampf" hatte sich Hitler schon in zwei längeren Exkursen dazu geäußert. Auch im zweiten Buch haben sich seine Grundgedanken dazu nicht allzu sehr verändert. ${ }^{58}$ Hitlers Pläne für die Eroberung von „Lebensraum" im Osten Europas setzten eine gewisse Bündnispolitik voraus. England und Italien waren in dieser Hinsicht für ihn ideale Partner, um seine Ziele durchzuführen. Diese Bündnisse würden einerseits gegen den „Erzfeind“ Frankreich gerichtet sein und einer deutschen Besetzung osteuropäischer Gebiete - auf Kosten der Sowjetunion - nicht im Wege stehen. Das faschistische Italien als Bündnispartner zu gewinnen, war für Hitler ein besonderer Kernpunkt seiner außenpolitischen Pläne. Aus diesem Grund legte er im letzten Kapitel auf 76 Seiten ausführlich dar, wieso Italien und Deutschland die idealen Voraussetzungen für ein zukünftiges Bündnis aufwiesen.

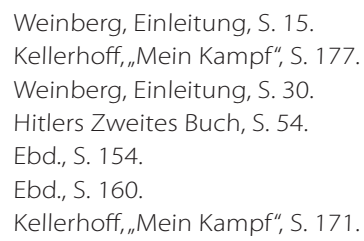


Weinberg hat sich auch mit der Klärung über das genaue Datum der Niederschrift beschäftigt und ist einigen Indizien nachgegangen, vom Inhalt des Buches selbst ausgehend und von Überlieferungen von Hitlers Weggefährten. Hitler greift beispielsweise in seinem Buch den seit 1923 amtierenden deutschen Außenminister Gustav Stresemann wegen seiner liberalen Außenpolitik, besonders wegen seiner Ausgleichspolitik mit Frankreich, an. Stresemann starb 1929, weshalb der Zeitpunkt der Niederschrift des Manuskriptes noch vor dessen Tod angelegt werden darf. ${ }^{59}$ Zudem ist von der französischen Besetzung des linken Rheinufers ${ }^{60}$ im Manuskript die Rede, die 1930 beendet wurde, sowie von dem Dawes-Plan, der die Zahlung der Reparationsschulden Deutschlands aus dem Ersten Weltkrieg regelte: sein Nachfolger, der Young-Plan, wurde erst 1929 ausgehandelt. ${ }^{61}$ Mehrere Bemerkungen zu alltagspolitischen Geschehnissen, zitierten Artikeln und eine große Ähnlichkeit zu einer Rede, die er am 13. Juli hielt, weisen auf den Sommer 1928 als Entstehungszeit der Schrift hin. ${ }^{62}$ Zudem gibt es auch in Briefen von Hitlers engsten Gefährten, wie Rudolf Heß, Hinweise darauf, dass Hitler im Sommer des Jahres 1928 ein neues Buch über die Außenpolitik geschrieben hat. ${ }^{63}$

Der Anlass, zu dem Hitler sich verleitet fühlte, dieses zweite Buch zu schreiben, wird schon von ihm selbst im Vorwort angesprochen. Im zweiten Band von "Mein Kampf" und in einer Sonderbroschüre von 1926 hatte sich Hitler intensiv mit der sogenannten Südtirol-Frage auseinandergesetzt. Seinen eigenen Worten nach konnte er außerhalb des nationalsozialistischen Lagers kaum Anhänger für seine Bündnispläne bezüglich Italiens finden. In den letzten Jahren hätte sich sogar die „Hetze“ seiner Gegner gegen seine Ansichten verstärkt, weshalb er sich verantwortlich fühle, die nationalsozialistische Außenpolitik näher auszuführen und die Leserschaft von seiner "Richtigkeit" zu überzeugen. ${ }^{64}$

Es liegt nahe, sich deshalb etwas genauer mit der Situation des Jahres 1928 zu beschäftigen, in der Hitler es für nötig befand, ein eigenes Buch über sein außenpolitisches Programm zu schreiben.

\subsection{Reichstagswahl und die Südtiro/-Frage 1928}

Am 20. Mai 1928 fanden in Deutschland Reichstagswahlen statt. Die NSDAP erhielt dabei nur 2,6 Prozent, was in etwa 810.000 Stimmen entsprach. Im Reichstag waren sie damit mit zwölf Sitzen vertreten; eine Wahlniederlage für Hitler und seine Bewegung. ${ }^{65}$ In den Wochen des Wahlkampfes machte vor allem ein Thema der NSDAP und deren Presseorgane zu schaffen: Die Südtirol-Frage.

Südtirol, seit Ende des Ersten Weltkrieges von Italien annektiert, war für Hitler kein unbekanntes Thema. Schon seit 1922 hatte er sich des Öfteren in Reden zu Südtirol

59 Weinberg, Einleitung, S. 20.

60 Hitlers Zweites Buch, S. 148

61 Ebd., S. 174

62 Weinberg, Einleitung, S. 20.

63 Plöckinger, Geschichte eines Buches, S. 162.

64 Hitlers Zweites Buch, S. 43-45.

65 Kellerhoff, "Mein Kampf", S. 177. 
geäußert. Quer durch alle Parteien Deutschlands wurde die Ansicht vertreten, die Abtretung Südtirols mit seiner vorwiegend deutschsprachigen Bevölkerung müsse rückgängig gemacht werden. Die einzige Ausnahme bildete dabei, und das mag auf den ersten Blick befremdlich erscheinen, die NSDAP. Hitler beharrte auf der Notwendigkeit eines Bündnisses mit dem faschistischen Italien. Das Thema war Hitler anscheinend so wichtig, dass er ein unfertiges Kapitel des zweiten Bandes von "Mein Kampf“ vorab im Februar 1926 als Sonderbroschüre unter dem Titel "Die Südtiroler Frage und das deutsche Bündnisproblem“ in 10.000 Exemplaren drucken ließ.6 Hitlers Bewunderung für den "genialen Staatsmann Benito Mussolini"67 und seine Aussagen über den notwendigen Verzicht von Südtirol stießen bei der deutschen Bevölkerung jedoch auf wenig Verständnis. Entgegen seiner ideologischen Ansichten zu Deutschen im Ausland nahm er den Verbleib des kulturell und historisch deutsch geprägten Südtirols bei Italien bewusst in Kauf.

Eine neue Brisanz erhielt die Südtirol-Frage im Wahljahr 1928, als die "Italianisierung" in Südtirol im schulischen Bereich einen neuen Höhepunkt erreichte. Das Schulgesetz von 1923, die Lex Gentile, sollte dafür sorgen, dass in den deutschsprachigen Schulen nur mehr in italienischer Sprache unterrichtet wurde. Die bewusste Absicht hinter diesem Gesetz war die "Zerstörung der deutschen Schule"68. Der einzige Bereich in dem noch in deutscher Sprache unterrichtet werden durfte, war der Religionsunterricht. 1928 plante die italienische Regierung aber auch diesen Bereich zu „italianisieren“, was in den österreichischen und deutschen Medien eine Welle von empörten Reaktionen auslöste. Hitler und das Sprachorgan seiner Partei, der "Völkische Beobachter", sahen sich nun der mühsamen Aufgabe gegenüber, Mussolini und sein Verhalten zu verteidigen. Besonders im Wahlkampf kamen er und seine Anhänger in Bedrängnis, ihre Haltung bezüglich Südtirol, angesichts des rigorosen Vorgehens der italienischen Regierung, zu rechtfertigen. ${ }^{69}$

Hitler, dessen großes Vorbild Mussolini - und damit der einzige europäische Regierungschef mit einer ähnlichen ideologischen Ausrichtung wie er selbst - war, beschuldigte lieber die deutsche Presse, Juden, Kommunisten sowie gegnerische Parteien das Thema Südtirol für ihre jeweils eigenen Zwecke zu missbrauchen. ${ }^{70}$ Im Wahlkampf hielt sich Hitler dann auch auffallend zurück, wenn es um das Thema Südtirol ging. Der Widerspruch in seinen Positionen, einerseits der Anspruch, dass die im Ausland lebenden Deutschen zum Vaterland gehörten, und andererseits die Ausnahme, dass diese Regel für die deutschen Südtiroler nicht gelten würde, bot den politischen Gegnern Hitlers eine perfekte Zielscheibe im Wahlkampf.

Am Wahltag gipfelte diese politische Schlammschlacht in einer Plakataktion der Münchner SPD. Auf den Anzeigen wurde Hitler vorgeworfen, er habe durch finanzielle Unterstützung Mussolinis im Wahlkampf Südtirol und seine Bewohner verkauft. Ob-

66 Kellerhoff, „Mein Kampf“, S. 174

67 Hitlers Zweites Buch, S. 182.

68 Rolf Steininger, Südtirol. Vom Ersten Weltkrieg bis zur Gegenwart, Innsbruck 2003, S. 26.

69 Kellerhoff, "Mein Kampf", S. 175-176.

70 Hitlers Zweites Buch, S. 191. 
wohl Hitler Anzeige erstattete und später vor Gericht auch Recht bekam, war der Schaden für die Partei und ihren Führer nicht mehr abzuwenden. ${ }^{71}$ Die Wahlniederlage war für Hitler wohl Anlass genug, sich für einige Wochen im Sommer aus der Öffentlichkeit nach Berchtesgaden zurückzuziehen und seine außenpolitischen Vorstellungen, besonders was die Beziehungen zu Italien anbelangte, in einem zweiten Buch verständlicher, vor allem aber für seine Leserschaft nachvollziehbarer vorzustellen. ${ }^{22}$

\subsection{Hitlers außenpolitische Pläne}

Im Vorwort begründet Hitler seine Arbeit an diesem Buch damit, dass die Südtirol-Frage von seinen Gegnern benutzt werde, um vor allem gegen die nationalsozialistische Bewegung und seine Person zu hetzen. Da sich die Angriffe und Animositäten gegen Italien verstärkt hätten, sehe er sich nun gezwungen seine Bündnispläne, an denen er nach wie vor festhalte, gründlicher darzustellen. Dabei erkennt er selbst, dass er für seine Pläne mit Mussolini-Italien im gegenwärtigen Deutschland keine breite Mehrheit finden würde:

„So, wie schon öfter in anderen Dingen steht heute die nationalsozialistische Bewegung in ihrer außenpolitischen Einstellung innerhalb des deutschen Volkes und seines politischen Lebens vollkommen vereinsamt und vereinzelt dar."13

Trotzdem sieht Hitler nach wie vor ein Bündnis mit Italien als ein wichtiges Mittel für den Wiederaufstieg Deutschlands an. Im letzten und längsten Kapitel begründet Hitler sodann seine Anschauungen, wieso sich Italien als ein gleichwertiger und wichtiger Verbündeter einer nationalsozialistischen Außenpolitik eignen würde.

In erster Linie würden beide Staaten die gleichen Interessen, in Bezug auf ihre außenpolitischen Ziele, verbinden. Italien stehe, genau wie Deutschland, vor dem zukünftigen Problem einer Bevölkerungszunahme ohne ausreichend Raum zum Leben zur Verfügung zu haben. Während sich Deutschland also seinen Expansionsplänen im Osten und Norden Europas widmen könnte, würde Italien sich, ähnlich dem antiken Rom, vielmehr dem Mittelmeerraum zuwenden. Damit bestünde für beide Staaten kein Interessenskonflikt und der eine würde die Pläne des anderen keinesfalls behindern. ${ }^{74}$

Die Expansion Italiens in den Mittelmeerraum würde das Land, nach Hitlers Vorstellungen, unweigerlich in Konflikt mit Frankreich bringen, das seine eigene Vorherrschaft in diesem Gebiet bedroht sehen würde. Dieses außenpolitische Vorgehen würde somit eine "natürliche Feindschaft"75 zwischen den beiden Staaten noch vertiefen und Italien zugleich enger an Deutschland binden. Die zukünftigen Gegner eines Krieges sah Hitler also genauso wie schon seine zukünftigen Verbündeten voraus.

Kellerhoff, "Mein Kampf”, S. 176.

Plöckinger, Geschichte eines Buches, S. 162.

Hitlers Zweites Buch, S. 44.

Ebd., S. 176-179.

Ebd., S. 178. 
Der Eintritt Italiens auf Seiten der Entente im Ersten Weltkrieg war für Hitler ebenso wenig ein Hindernis für ein zukünftiges Bündnis zwischen den beiden Völkern. Der Grund, an dem von deutscher Seite wahrgenommenen „Verrat“ Italiens, lag nach Hitler an der damaligen Fehlentscheidung des deutschen Reiches, die habsburgischen Interessen der k.u.k. Monarchie, anstatt der eigenen zu verteidigen. ${ }^{76}$

Das nachdrücklichste Argument gegen eine Verbindung mit Italien, der Verzicht Südtirols, wollte Hitler genauso wenig gelten lassen. Für eine Verständigung mit Mussolini musste dieses Problem für die Leserschaft aber zumindest relativiert werden. Hitlers ersten Schuldigen fand er in der Presse, die nach dem Machtaufstieg Mussolinis das Thema Südtirol in extremster Weise überzogen dargestellt hätte. ${ }^{77}$ Die Menschen, die sich in Deutschland wegen der Unterdrückung der Südtiroler aufregten und sich nationale Patrioten nannten, waren für Hitler ausnahmslos Heuchler und ihre Erregung sei ohnehin nur gespielt. ${ }^{78}$ Die Schuld an den Ausschreitungen, die es gegen Südtiroler von Seiten des faschistischen Regimes gab, lag für Hitler bei denjenigen, die sich versuchten in inneritalienische Angelegenheiten einzumischen und in den Südtirolern sinnlose Hoffnungen zu wecken. ${ }^{79}$

Den Vorwurf der SPD am Wahltag, Hitler habe Südtirol verkauft, scheint er bei der Niederschrift dieses Kapitels noch nicht wirklich verwunden zu haben. In 14 Punkten zählt er nämlich die Umstände und Personen auf, die seiner Meinung nach für den Verlust Südtirols verantwortlich waren. Darin sieht er die Schuldigen vor allem in den Reihen seiner eigenen politischen Gegner. In typisch nationalsozialistischer Rhetorik schreibt Hitler:

„13. [Punkt] Südtirol wurde verloren und verraten durch die schamlosen Handlungen der Männer und Parteien, die nach dem Zusammenbruche die deutsche Ehre besudelten, das Ansehen unseres Volkes vor der Welt vernichteten und damit erst den Mut zu der Größe der Forderungen bei unseren Gegnern erweckten. Es wurde weiter verloren durch die erbärmliche Feigheit der national-bürgerlichen Parteien und vaterländischen Verbände, die vor dem Terror der Gemeinheit und Niedertracht überall ehrlos kapitulierten..$^{80}$

Die Südtiroler, Hitler variiert hinsichtlich der Bevölkerungszahlen von 164.000 200.000 ${ }^{81}$, hätten ihrerseits die Aufgabe in Hitlers ambitionierten Bündnisplänen eine „Brücke aufrichtiger gegenseitiger Verständigung zu bilden" ${ }^{\prime 2}$. Wie weit dieses Vorhaben von der damaligen herrschenden Realität abwich, schien Hitler bewusst nicht zur Kenntnis genommen zu haben.

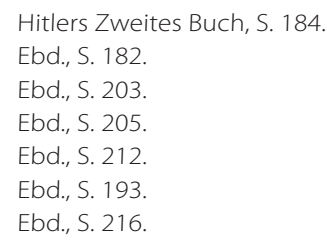


Bekanntermaßen wurde das Südtirol-Problem durch das Hitler-Mussolini Abkommen von 1939 "gelöst". Die Südtiroler hatten die Möglichkeit entweder in das Deutsche Reich auszuwandern und sich in eroberte Gebiete geschlossen anzusiedeln oder im faschistischen Italien zu bleiben, das ihre Kultur und Sprache weiterhin unterdrückte. Die Option war eine der schwerwiegendsten Erfahrungen der Südtiroler im 20. Jahrhundert, welche die Gesellschaft, Dorfgemeinschaften und sogar Familien tief spaltete. ${ }^{83}$ Für Hitler war sie anscheinend ein durchkalkuliertes und annehmbares Opfer seiner eigenen außenpolitischen Pläne.

\section{Verwahrung des Manuskripts}

Nachdem nun die Entstehungsgeschichte und kurz auf den Inhalt des Manuskriptes eingegangen wurde, bleibt noch die Frage zu klären, warum Hitlers Zweites Buch nicht nach seiner Niederschrift gedruckt und verkauft wurde. Das Manuskript blieb Ende Juli 1928 ohne weiteren Korrekturen oder Anmerkungen erst einmal liegen. Hitler selbst hatte wohl noch bis Anfang des Jahres 1929 mit dem Gedanken einer Vollendung und Veröffentlichung seiner Schrift gespielt. ${ }^{84}$ Inzwischen erwiesen sich jedoch mehrere Konstellationen für eine weitere Publikation von Hitlers Ideen als äußerst ungünstig. Die Verkaufszahlen von „Mein Kampf“ erreichten im Jahr 1928 einen Tiefstand, ${ }^{85}$ zudem lagen noch tausende Restbestände auf Lager. Der Direktor des Franz-Eher Verlags, Max Amann, riet Hitler wahrscheinlich von einem weiteren Buch, das mit "Mein Kampf" konkurrieren würde, ab. ${ }^{86}$

Das Manuskript wies überdies auch keine allzu großen Abweichungen von Hitlers Ausführungen in "Mein Kampf" auf. Es blieb in den Grundsätzen konstant und bot somit keine neuartigen Ideen für die nationalsozialistische Bewegung. ${ }^{87}$ Auch die finanzielle Situation der Partei zu diesem Zeitpunkt erlaubte keinen weiteren kostspieligen Druck einer Schrift, die allen Erwartungen nach, keine große Leserschaft anziehen würde. ${ }^{88}$

Darüber hinaus musste noch die veränderte politische Lage im Jahr 1929 berücksichtigt werden. Im Sommer 1929 war die NSDAP mit der bürgerlichen Deutschnationalen Partei verbündet, die von Hitler in seiner Schrift öfters angegriffen wurde. ${ }^{89}$ Das Buch hätte auch aufgrund der Ereignisse in den folgenden Monaten in großen Teilen überarbeitet werden müssen. Im Jahr, in dem Gustav Stresemann starb, der Young-Plan - der Nachfolger des Dawes-Plans - ausgearbeitet wurde und die Weltwirtschaftskrise ihren verheerenden Lauf nahm, hätte Hitler zu einer Revision seines Erstentwurfs wohl kaum Zeit gehabt.

\footnotetext{
83 Steininger, Südtirol, S. 43.

84 Plöckinger, Geschichte eines Buches, S. 163.

85 Ebd., S. 162

86 Kellerhoff, "Mein Kampf", S. 188 -Vitkine, Hitlers „Mein Kampf”, S. 49

87 Kellerhoff, „Mein Kampf", ebd.

88 Plöckinger, Geschichte eines Buches, S. 161.

89 Kellerhoff, "Mein Kampf", S. 189
} 
Erst nach den Wahlerfolgen der NSDAP und den wieder ansteigenden Verkaufszahlen von "Mein Kampf" schlug der Verlag Hitler vor, nun auch seine zweite Schrift herauszugeben. Doch Hitler lehnte dieses Angebot ab..$^{90}$ Nach seiner Machtübernahme 1933 war Hitler wiederum darum bemüht seine außenpolitischen Pläne, die er Jahre zuvor entworfen hatte, dem Ausland nicht mehr zugänglich zu machen, selbst wenn man die Grundzüge noch immer in „Mein Kampf“ nachlesen konnte. Das Manuskript wurde also in den Tresor des Verlages eingeschlossen und wie Josef Berg angibt, sogar unter strengster Geheimhaltung aufbewahrt. ${ }^{91}$ Von einer völligen Geheimhaltung kann jedoch nicht die Rede sein, denn zumindest die engsten Hitlergefährten, sowie einige seiner Sekretärinnen wussten von dem Dokument. Hitler selbst sprach noch Jahre später in Andeutungen von einer zweiten unvollendeten Schrift zur Außenpolitik. ${ }^{92}$ Der Öffentlichkeit bekannt und zugänglich wurde sie allerdings erst durch die Arbeit von Historikern; dreißig Jahre nach ihrer Niederschrift.

\section{Fazit}

Hitlers Zweites Buch stellt für die NS-Forschung zweifellos ein wichtiges Dokument dar. Für Historikerlnnen, die sich näher mit Hitlers außenpolitischen Plänen beschäftigen, sieht diese Quelle wertvolle Einsichten, vor allem für die Zeit, als Hitler noch weit entfernt von jeglicher politischen Bedeutsamkeit war. Wenn auch vieles, das in dem Buch vorkommt, schon viel länger und wahrscheinlich öfters in "Mein Kampf" nachzulesen war, zeigt es doch in aller Deutlichkeit, wie konsequent und unnachgiebig Hitler in den Jahren vor seiner Machtergreifung an seinen Zukunftsplänen für Deutschland festhielt. Seine Bündnispolitik, die er in diesem zweiten Buch detailliert dargestellt hat, ist deshalb auch ein Zeugnis dieser konstanten und manchmal unrealistischen Weltsicht Hitlers.

Hitler schrieb in einer Zeitphase, die auf alles andere als eine glückliche Zukunft und das politische Überleben der Nationalsozialisten hinwies. Die Wahlniederlage von 1928 nahm Hitler als Anlass, seine außenpolitischen Vorstellungen in einem weiteren Werk auszuführen. Eine Streitfrage war das altbekannte Südtirol-Problem. Hitler und seine Bewegung standen mit der Haltung, für ein Bündnis mit Mussolini-Italien sei auf eine Rückforderung Südtirols zu verzichten, vollkommen isoliert da. Selbst für seine Anhänger musste diese Position hart zu verteidigen gewesen sein. Die Geschichte hat gezeigt, dass Hitler an seinem Pakt mit Mussolini und der Anerkennung der Brennergrenze, zumindest bis zum Einmarsch in Italien 1943 hartnäckig festhielt.

Die Gründe für eine Nichtveröffentlichung des Werks waren höchst wahrscheinlich mehrere: Fehlende finanzielle Mittel, schleppender Absatz von "Mein Kampf" und veränderte politische Umstände ließen wohl eine zeitraubende Überarbeitung des Manuskripts nicht zu. Nach der Machtergreifung hatte Hitler wohl kein großes Interesse mehr an einer weiteren Veröffentlichung seiner außenpolitischen Pläne. So wurde es dann im Tresor des Franz-Eher-Verlags bis zum Ende des Krieges weggeschlossen und

90 Vitkine, Hitlers „Mein Kampf“, S. 49.

91 Weinberg, Anlage, S. 225.

92 Plöckinger, Geschichte eines Buches, S. 164; Kellerhoff, "Mein Kampf", S. 190. 
konnte erst nach sorgfältiger Arbeit und Recherche von Gerhard L. Weinberg ans Licht gebracht und identifiziert werden. Die Reaktion auf die Publikation außerhalb des wissenschaftlichen Leserkreises hielt sich zumeist in Grenzen, was nicht verwundern darf. Für eine genauere Beschäftigung mit Hitlers weltpolitischen Ansichten griffen die Meisten doch noch zu dem allgegenwärtigen ersten Werk von Adolf Hitler: "Mein Kampf".

\section{Quellen}

Hitlers Zweites Buch. Ein Dokument aus dem Jahr 1928. Eingeleitet und kommentiert von Gerhard L. Weinberg. Mit einem Geleitwort von Hans Rothfels, (Quellen und Darstellungen zur Zeitgeschichte 7), Stuttgart 1961.

\section{Literatur}

Besson, Waldemar, Rezension zu: Hitlers zweites Buch. Ein Dokument aus dem Jahre 1928. Eingeleitet und kommentiert von Gerhard L. Weinberg. Mit einem Geleitwort von Hans Rothfels, Stuttgart 1961, in: Historische Zeitschrift, 197 (1963), Heft 3, S. 670-673, [http://www.jstor.org/stable/27613666], eingesehen 26.5.2016.

Brodkorb, Mathias, Hitlers Zweites Buch. Mit den europäischen Nationen gegen amerikanische Globalisierung?, Störungsmelder, Blogeintrag vom 21.5.2009, [http://blog. zeit.de/stoerungsmelder/2009/05/21/hitlers-zweites-buch-mit-den-europaischen-nationen-gegen-amerikanische-globalisierung_1077], eingesehen 29.8.2016.

Broszat, Martin, Betrachtungen zu Hitlers Zweitem Buch, in: Vierteljahrshefte für Zeitgeschichte 9 (1961), Heft 4, S. 417-429.

Enigma Books, Hitler's Second Book. The Unpublished Sequel to Mein Kampf, o. D., [http://www.enigmabooks.com/store/catalog/moreinfo.php?id=321], eingesehen 29.8.2016.

Johnson, Daniel, Revealed: the amazing story behind Hitler's second book, in: The Telegraph, 25.9.2003, [http://www.telegraph.co.uk/culture/books/3603289/Revealed-theamazing-story-behind-Hitlers-second-book.html], eingesehen 22.8.2016.

Kellerhoff, Sven Felix, "Mein Kampf". Die Karriere eines deutschen Buches, Stuttgart 2015.

Kritische "Mein Kampf" - Ausgabe wird zum Bestseller, in: Die Welt 27.2.2016, [http:// www.welt.de/kultur/article152709505/Kritische-Mein-Kampf-Ausgabe-wird-zumBestseller.html], eingesehen 25.8.2016.

Lebovic, Nitzan, Hitler in a Post-Ideological Age. The Sequel, in: H-German. H-Net Reviews, 2006, [http://www.h-net.org/reviews/showrev.php?id=11363], eingesehen 30.8.2016.

Neugebauer, Wolfgang, Rothfels, Hans, in: Neue Deutsche Biographie 22 (2005), S. 123-125 Onlinefassung: [https://www.deutsche-biographie.de/\%20pnd118749943. html], eingesehen 22.8.2016. 
Overy, Richard, Mein Kampf. The Sequel, in: The Guardian, 1.7.2003, [https://www. theguardian.com/books/2003/jul/01/news], eingesehen 30.8.2016.

Plöckinger, Othmar, Geschichte eines Buches: Adolf Hitlers "Mein Kampf“ 1922-1945, München $2011^{2}$.

Steininger, Rolf, Südtirol. Vom Ersten Weltkrieg bis zur Gegenwart, Innsbruck 2003.

Trevor-Roper, Hugh Redwald, Hitlers Kriegsziele, in: Vierteljahrshefte für Zeitgeschichte 8 (1960), Heft 2, S. 121-133.

Vitkine, Antoine, Hitlers „Mein Kampf“. Geschichte eines Buches, Hamburg 2015.

Weinberg, Gerhard L., in: Jahresberichte für Deutsche Geschichte, 22.12.2015, [http:// jdgdb.bbaw.de/cgi-bin/jdg?lang=de\&t_idn=\%28DE-588\%29105818925], eingesehen 22.8.2016.

Sabine Viktoria Kofler ist Studentin der Geschichtswissenschaften (MA) an der Universität Innsbruck im 5. Semester. sabine.kofler@student.uibk.ac.at

\section{Zitation dieses Beitrages}

Sabine Viktoria Kofler, Hitlers Zweites Buch - Eine vergessene Quelle der Zeitgeschichte zu Hitlers außenpolitischen Ansichten, in: historia.scribere 9 (2017), S. 271-286, [http:// historia.scribere.at], 2016-2017, eingesehen 14.6.2017 (=aktuelles Datum).

Creative Commons Licences 3.0 Österreich unter Wahrung der Urheberrechte der Autorlnnen. 